\title{
Régénération osseuse guidée dans le secteur antérieur
}

\section{Guided bone regeneration in the anterior sector}

\section{Résumé}

\section{MOTS-CLEFS :}

- Régénération osseuse guidée, os autogène, substituts osseux, membrane, reconstitution défaut osseux

\section{KEYWORDS:}

- Guided bone regeneration, autogenous bone, bone substitutes, membrane, bone defect reconstitution

AOS $n^{\circ} 288-2018$
La régénération osseuse guidée est aujourd'hui une solution fiable pour la reconstitution des défauts osseux antérieurs. Les biomatériaux actuels, en particules, mélangés avec de l'os autogène, protégés par une membrane en collagène soutenue par des «tenting screws" nous permettent de traiter des défauts osseux importants, évitant ainsi l'utilisation des blocs osseux dont le prélèvement est très invasif, l'adaptation aux contours du défaut osseux difficile et la résorption par manque de vascularisation importante.
Abstract

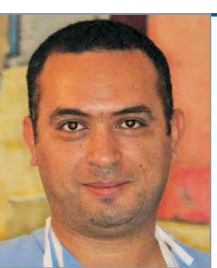

Kilani BOUSSETTA, Exercice exclusif en implantologie et chirurgie buccale, 2 bis rue d'argentine 1002 Tunis, le belvédère, Tel : (00216)53218715email : boussettakilani@yahoo.fr

Sarra JAOUA, Exercice exclusif en orthopédie dento-faciale de l'enfant et de l'adulte, Le belvédère Médical, 78 Avenue de la liberté, 1002 Tunis le belvédère.
$\mathrm{L}$ es défauts osseux sont aujourd'hui une entrave a une réhabilitation implanto-portée esthétique et fonctionnelle, ces défauts peuvent être d'origine traumatique, infectieuse, tumorale ou des défauts congénitaux (1)

La régénération osseuse guidée, également appelée "régénération osseuse protégée par une membrane » est une technique fiable qui a montré des résultats prédictibles avec des taux de succès importants, cette technique fait appel a des membranes barrières pour séparer deux compartiments : un compartiment osseux formé essentiellement par le défaut osseux, l'os, la cavité médullaire et un compartiment de tissus mous formé par la gencive sus jacente et le périoste (2).

Cette barrière mécanique va empêcher le passage des cellules non ostéogènes provenant des tissus mous, le défaut osseux ainsi protégé va être comblé par les vaisseaux osseux et les cellules ostéogéniques provenant de la cavité médullaire (2-3).
Durant la cicatrisation plusieurs composants cellulaires ayant des vitesses de migration différentes vont entrer en jeu, la membrane va ainsi retarder l'invasion du défaut par les cellules inhibitrices, beaucoup plus rapides a envahir le défaut (4).

Seibert et nyman démontrent une parfaite reconstruction osseuse avec de l'os néo-formé d'un défaut vestibulo-lingual induit chirurgicalement après 90 jours (5).

Buser et coll (1995) rapportent un gain de 1,5 et $5,5 \mathrm{~mm}$ de hauteur avec de l'os néo-formé 10 mois après une régénération osseuse guidée (6).

Il est actuellement admis que le taux de succès des implants posés dans de l'os regenéré avec les techniques de régénération osseuse guidée est similaire a ceux posés dans des sites non augmentés, Christoph et coll dans une revue systématique rapportent des résultats avec des taux de survie de $79,4 \%$ a $100 \%$ (7-8-9). 
Clementini rapporte que les implants posés après une régénération osseuse guidée sont plus prédictibles que ceux posés en même temps que la ROG (10-11-12). Plusieurs auteurs accordent plus d'importance au rôle protecteur du caillot sanguin par la membrane par rapport a l'invasion et la compétition cellulaire (13). Le rôle de la membrane dans la ROG est primordial, puisque c'est elle qui maintient l'espace, cette dernière doit être bien tolérée par les tissus environnants sans induire de réactions inflammatoires. Pour assurer la fonction barrière, des membranes non résorbables ont été initialement employées avec des matériaux différents, les membranes en poly-tetrafluoroéthyl (PTFE) étaient les plus utilisées en chirurgie parodontale (Figure 1), Lang et coll dans une étude rétrospective dans le but de démontrer l'efficacité des membranes non résorbables dans la reconstructions des défauts osseux de morphologies différentes, constatent une néoformation osseuse dans $90 \%$ a $100 \%$ de l'espace disponible sous la membrane après 6 à 8 mois de l'intervention (14-15), mais en cas d'exposition le volume de cette néoformation est très compromis. La nécessité d'un deuxième temps chirurgical et le risque d'exposition important entrainant l'échec de la régénération osseuse guidée a abouti à l'utilisation de membranes résorbables, ces derniers offrent les mêmes résultats mais présentent plusieurs avantages parmi lesquels on cite une bio-compatibilité élevée et une meilleure maniabilité (16).

Plusieurs matériaux ont été aussi utilisés, on distingue a ce jours deux types de membranes, les membranes naturelles composé de collagène (Figure 2) ou de chitosan et les membranes synthétiques aliphatic polyesters, primarily poly (L-lactide) (PLLA) et poly (L-lactide-co-glycolide) (PLGA) co-polymers (4).

Dans une étude comparative Vierra constate que les membranes synthétiques présentent un taux d'exposition plus important que les sites greffés et protégés par une membrane en collagène (50\% des sites contre $12,5 \%$ des sites) (17).

Les membranes collagènes sont très documentés, elles présentent plusieurs avantages parmi lesquels on cite l'accélération de la cicatrisation muqueuse, une bonne intégration avec le tissu conjonctif, un

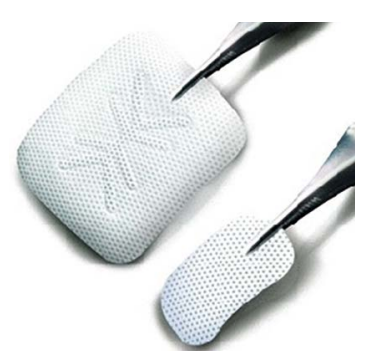

Figure 1.

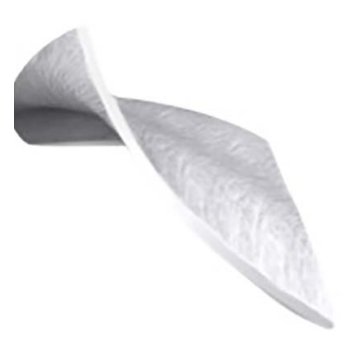

Figure 2. pouvoir important de prolifération et de fixation pour les cellules osteoblastiques et fibroblastiques (2-4-18). Le problème majeur des membranes résorbables est le temps de résorption rapide et aléatoire, Herten dans une étude explorant la dégradation des membranes en collagène montre une diminution de l'épaisseur de la membrane de $50 \%$ après une période de 4 semaines (19), ce temps de résorption varie avec le $\mathrm{PH}$ du site et la composition de la membrane, (généralement de 4 à 12 semaines).

Pour augmenter la fonction barrière certains auteurs préconisent la technique en double épaisseur (20).

Plusieurs paramètres concernant la membrane affectent la régénération osseuse comme la composition de la membrane, l'épaisseur de la membrane, la porosité et la taille des pores (4).

Il est actuellement admis que les membranes avec des macropores facilitent la régénération osseuse guidée plus que les membranes avec des micropores (18).

Konstantinos met en évidence le rôle crucial du périoste dans la régénération osseuse guidée, ainsi le périoste est formée par deux couches : une interne formée de capillaires sanguins, de nerfs, et de cellules mésenchymateuses capables de se différencier en cellules ostéoblastiques qui sont sources de facteurs de croissance jouant un rôle très important dans le mécanisme de cicatrisation et de remodelage a la surface corticale de l'os. La couche externe est fibreuse, elle contient des fibroblastes, des vaisseaux et des fibres de Sharpey, la surface du périoste est riche en cellules mésenchymateuses multipotentes en combinaison avec des facteurs de croissances qui sont secrétés après une blessure du périoste contribuant ainsi au processus de cicatrisation osseuse (21).

Dans la zone esthétique, l'utilisation d'un biomatériau a faible taux de substitution est très important dans le sens ou un volume osseux facial est primordial pour le maintien des tissus mous (22), l'os déprotéinisé d'origine bovine reste à ce jour, le matériau le plus documenté. C'est un bio-matériau ostéoconducteur, il favorise l'adhésion cellulaire, la cicatrisation et la formation osseuse, il est très proche de l'os humain de part sa structure physique et chimique. Pour Simion un mélange 1:1 d'os d'origine bovine avec de l'os autogène peut être utilisé pour la régénération osseuse verticale, l'os néoformé aboutit a l'ostéointegration des implants mis en même temps ou après 6 mois de la greffe osseuse, les études histologiques montrent une très lente résorption de l'os d'origine bovine et son remplacement par une néoformation osseuse (2324-25-26-27). Dans une étude comparative Broggini Nina compare le potentiel ostéogénique et le taux de substitution de l'os autogène avec 3 biomatériaux (Os déprotéinisé d'origine bovine, calcium phosphate 
bi-calcique, hydroxyapatite nanocrystallin), il constate que l'os autogène présente le meilleur taux de néoformation osseuse contrairement aux autres (28).

Selon les travaux de Karageorgiou une porosité du biomatériau supérieur de $300 \mu \mathrm{m}$ favorise l'ostéogenèse, les granules sont capable d'être colonisées par les vaisseaux et d'être bien oxygénée (29). Klawitter et Hulbert confirment cette information en démontrant que la taille minimale des pores compatibles avec la formation d'os minéralisé est d'environ $100 \mu \mathrm{m}$, celle nécessaire pour la formation de structures semblables aux ostéons est de $200 \mu \mathrm{m}$ (30). Karageorgiou constate que la nature de l'os néoformé dépend de la nature du biomatériau et de la géometrie des pores (24).

Le caillot sanguin fournit la fibrine qui est la matrice de la cicatrisation osseuse, l'os autogène en copeaux joue le rôle d'armature pour l'os néoformé, il est aussi source de cellules souches ostéogènes et de facteur de croissance et de différenciation qui se lient chimiquement aux protéines de la fibrine, les cellules précurseurs vont migrer a la surface de l'os et libérer les protéines morphogénétiques osseuses fixés par l'intermédiaires des enzymes spécifiques (2-31).

Pour Hellem et coll. l'utilisation de l'os autogéne en combinaison avec des copeaux d'os déprotéinisé d'origine bovine avec un ratio 50/50 stabilisé avec de la fibrine pour l'augmentation osseuse horizontale de la crête démontre une stabilité implantaire avec un taux de survie implantaire élevé, le suivi de 74 implants montre une résorption osseuse marginale moyenne pour une période de 3 ans de $0,3 \pm 0,2 \mathrm{~mm}(32-33)$.
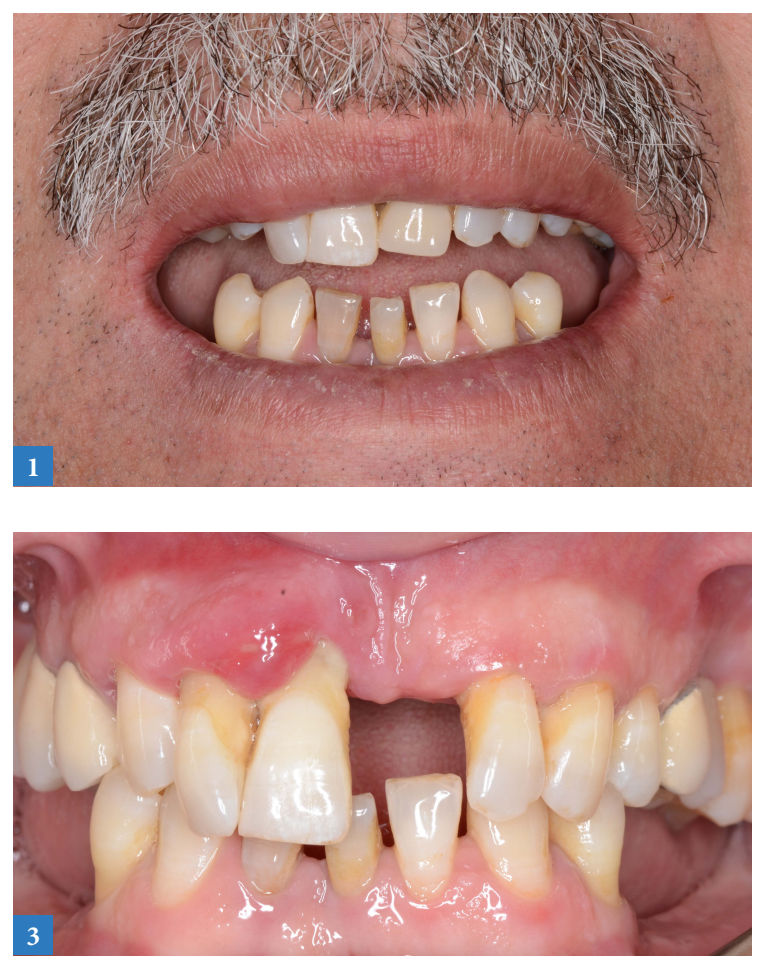

La régéneration osseuse guidée peut être altérée par le manque de vascularisation du site, l'instabilité mécanique des vis, la contamination du greffon après déhiscence de la membrane et la résorption rapide de la membrane vu les faibles caractéristiques mécaniques des membranes en collagène (33). Des vis de tentes «tenting screws» vont permettre de maintenir un espace suffisant pendant la cicatrisation (34), aidant ainsi a la stabilisation du caillot sanguin, les vis sont disponibles en plusieurs longueurs : $7 \mathrm{~mm}, 10 \mathrm{~mm}$, $13 \mathrm{~mm}, 15 \mathrm{~mm}$, un forage précède la mise en place de la vis.

Monsieur L. K., âgé de 41 ans est adressé a notre cabinet pour un traitement implantaire dans le secteur antérieur, le patient est en bonne santé et non tabagique.

L'incisive centrale maxillaire gauche (21) a été perdue suite a une maladie parodontale.

L'examen clinique pré-opératoire avec évaluation du risque esthétique a révélé deux facteurs de risques : la position haute de la lèvre supérieure et la ligne gingivale marginale non harmonieuse.

Après discussion avec le patient, ce dernier donne son accord pour le plan de traitement suivant :

traitement parodontal ;

I extraction des dents 12-11;

$\checkmark$ augmentation osseuse par la méthode de la régénération osseuse guidée du secteur antérieur ;

$\checkmark$ six mois après, pose des implants au niveau des sites 12 et 21 ;

I six mois après la pose des implants, réhabilitation prothétique du secteur.
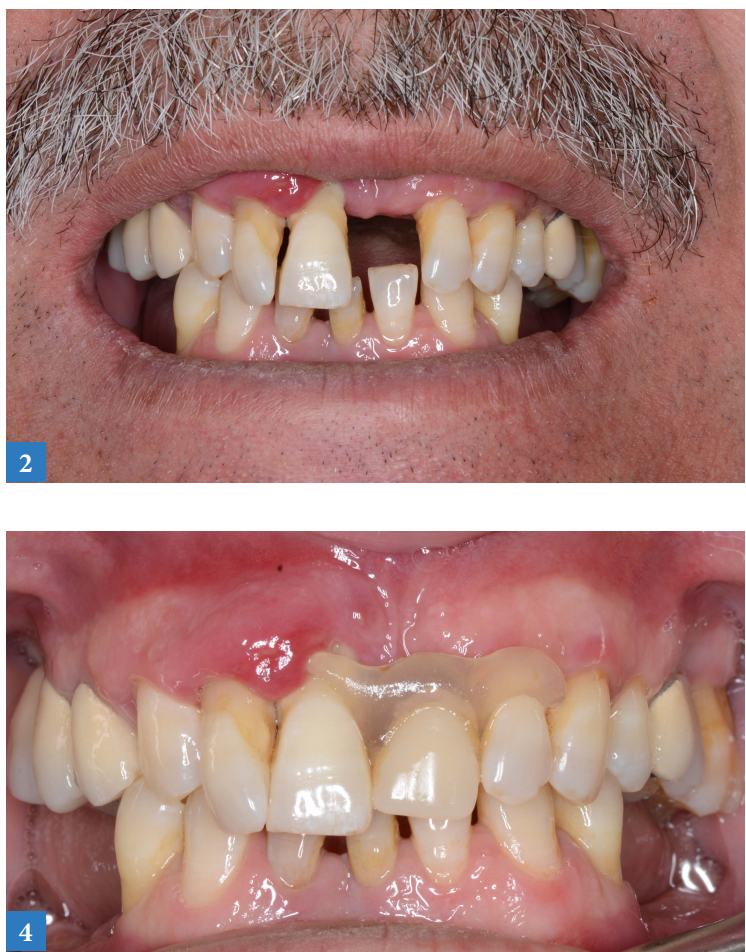

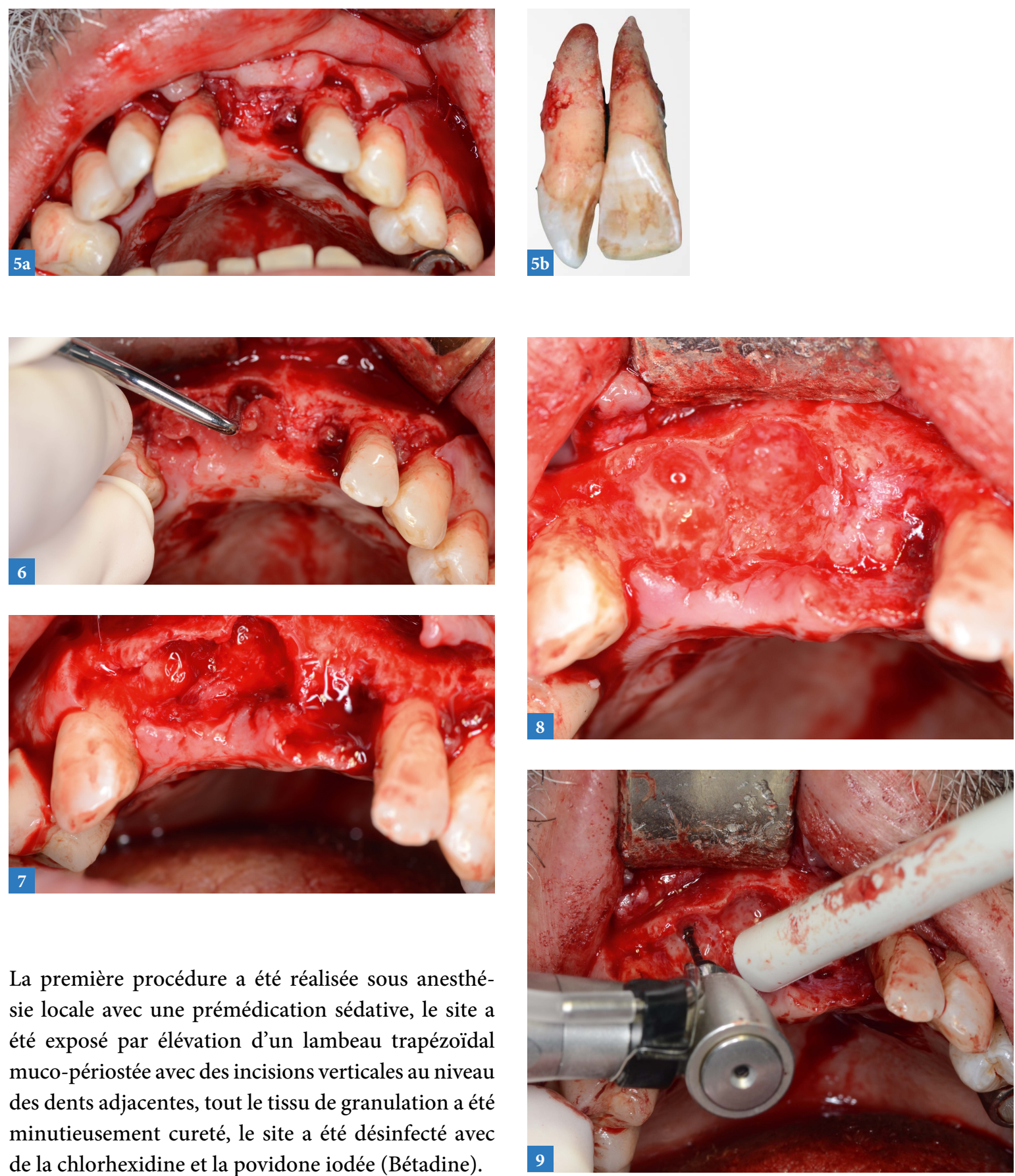

La première procédure a été réalisée sous anesthésie locale avec une prémédication sédative, le site a été exposé par élévation d'un lambeau trapézoïdal muco-périostée avec des incisions verticales au niveau des dents adjacentes, tout le tissu de granulation a été minutieusement cureté, le site a été désinfecté avec de la chlorhexidine et la povidone iodée (Bétadine). La phase d'assainissement a mis en évidence un large défaut horizontal et vertical.

Afin de permettre au défaut osseux d'être envahi par des néo-vaisseaux sanguins, permettant ainsi une bonne irrigation du bio-matériau, la corticale osseuse a été soigneusement perforée en plusieurs endroits, deux vis de tente ont été posées dans les sites les plus déclives du défaut afin d'éviter l'effondrement de la membrane de collagène et assurer ainsi la stabilité du greffon.

La hauteur des vis a été choisie de telle sorte que la tête de vis coïncide avec le niveau de l'os au niveau des dents collatérales au défaut et avoir une pénétration de $4 \mathrm{~mm}$ minimum au niveau de l'os pour avoir une bonne stabilité des vis, les vis ont un état de surface lisse afin de permettre leur dévissage lors

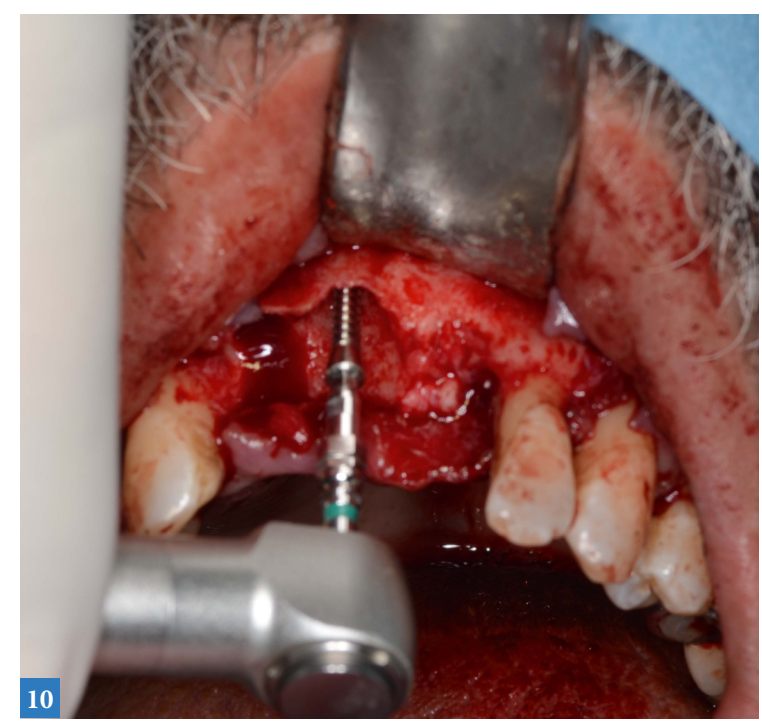



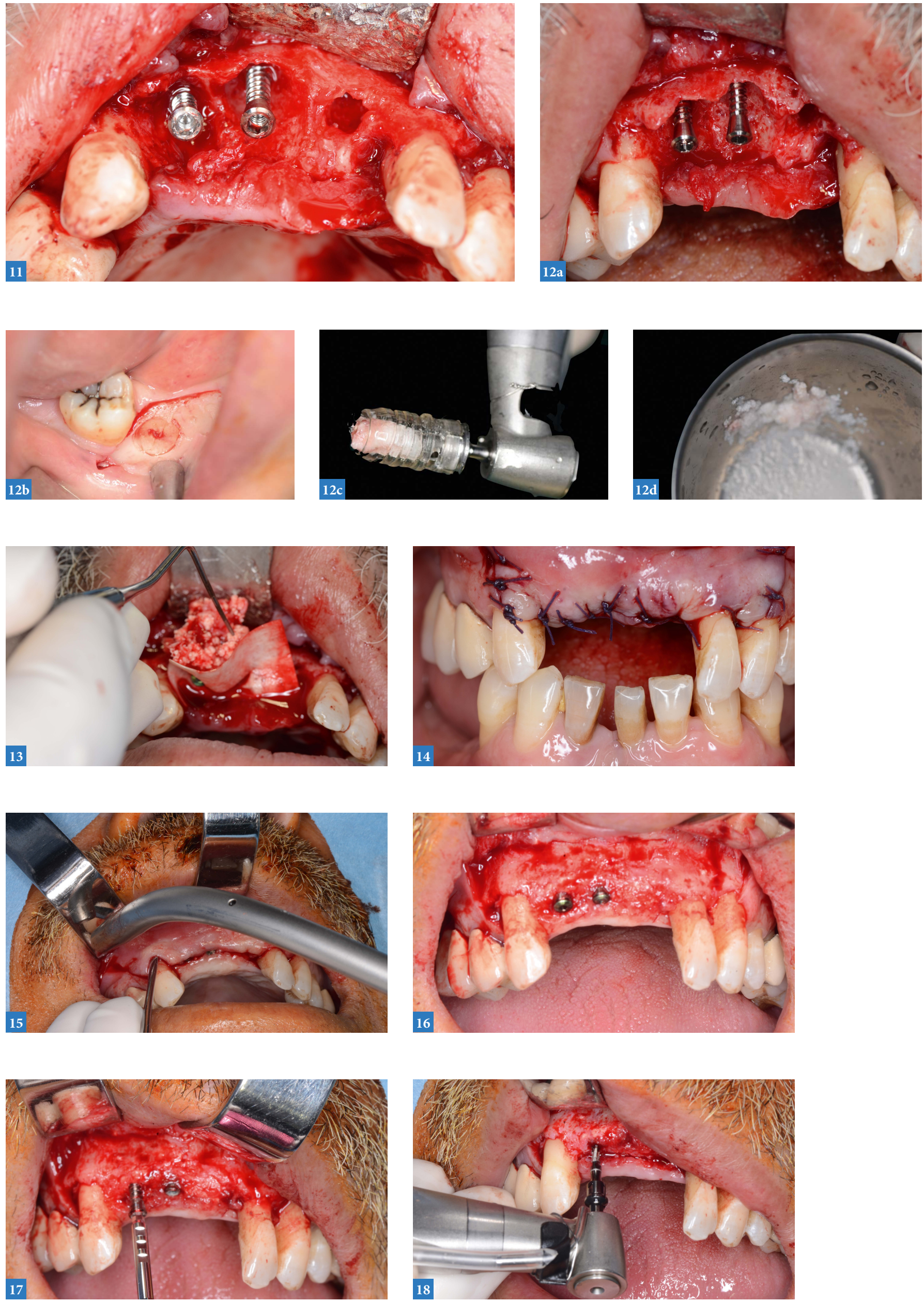


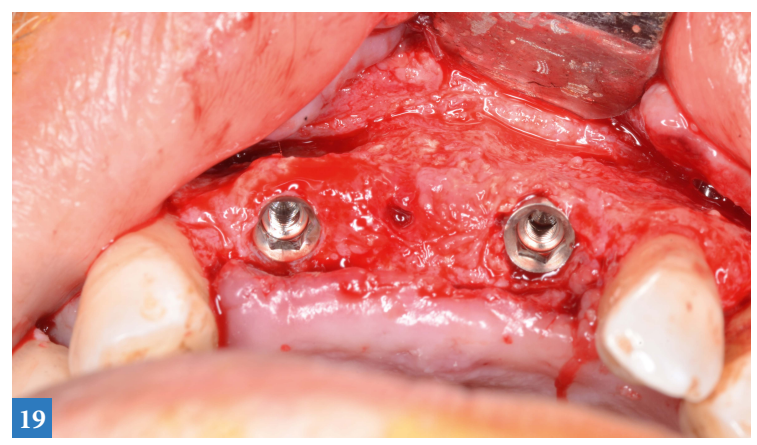

du deuxième temps chirurgical, lors de la pose des implants dentaires.

Le prélèvement de particules osseuses autogènes est réalisé en ambulatoire au niveau de la zone molaire inférieure sous anesthésie locale, un foret ACM (auto schip maker) de neobiotech de $5 \mathrm{~mm}$ est utilisé a une vitesse de 250 tour par minute, un stop empêche le foret de pénétrer plus de $4 \mathrm{~mm}$ dans l'os, l'os ainsi prélevé est mélangé avec des particules d'os déprotéinisé d'origine bovine selon les proportions 50 \%-50 \%. Le mélange ainsi obtenu est mis au niveau du défaut

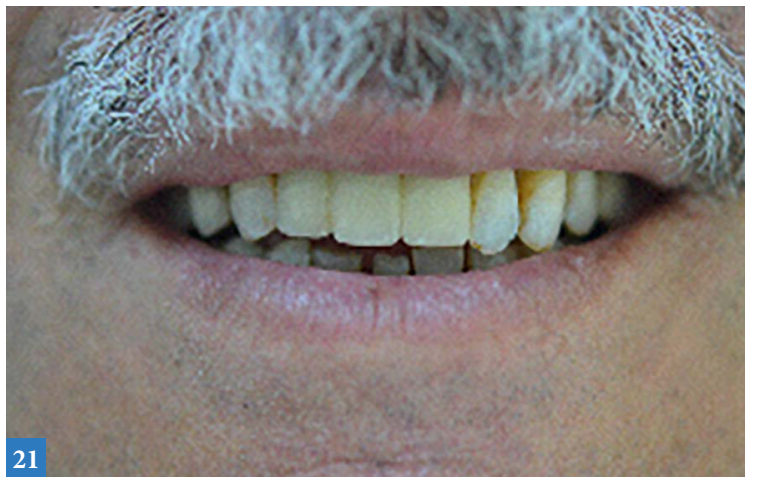

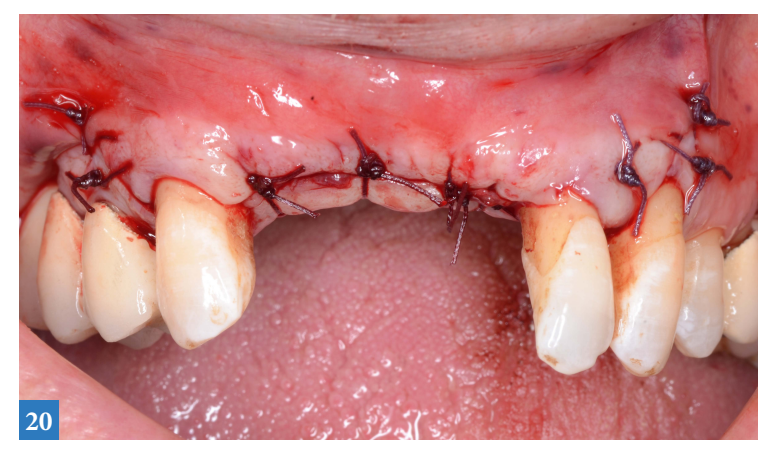

osseux entre les vis et sous la membrane de collagène qui a été fixé avec les vis de fixation, il faut éviter un tassement excessif du matériau afin de laisser la place pour la néo-vascularisation. une fermeture du lambeau sans tension est réalisée.

Le deuxième temps chirurgical commence 6 a 8 mois après le premier, on observe une néoformation osseuse compatible avec la mise en place de deux implants dentaires dans les sites 12 et 21 .

Six mois après le deuxième temps chirurgical, la restauration prothétique est réalisée.

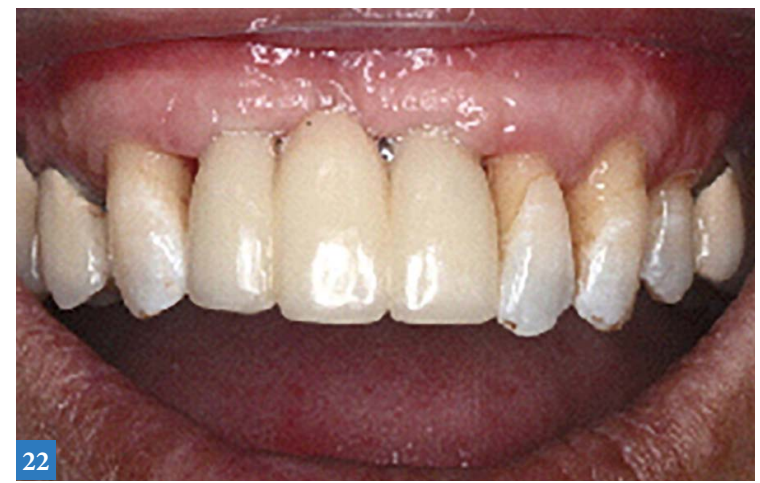

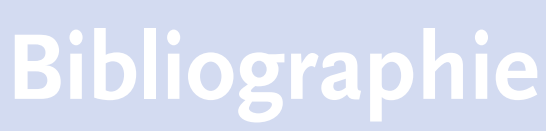

[1] Christoph HF, Hämmerle CH, Jung RE. Bone augmentation by means of barrier membranes. Periodontology 2000 2003; 33: 36-53.

[2] ITI Treatment Guide - Volume 7. Ridge augmentation procedures in implant patient. A staged approach. Quintessence edit, 2014.

[3] Simion M, Scarano A, Gionso L, Piatelli A. Guided bone regeneration using resorbable and nonresorbable membranes: A comparative histologic study in humans. Int J Oral Maxillofac Implants 1996; 22: 735-742.
[4] Dimitriou R, Mataliotakis G, Maria Calori G, Giannoudis PV. The role of barrier membranes for guided bone regeneration and restoration of large bone defects : current experimental and clinical evidence. $B M C$ Medicine 2012;10:81-9.

[5] Seibert J, Nyman S. Localized ridge augmentation in dogs: pilot study using membranes and hydroxyapatite. J Periodontol 1990; 61(3): 157-165.

[6] Buser D, Bragger U, Lang NP, Nyman S. Regeneration and elargment of jaw bone using guide bone regeneration. Clin Oral Implants Res 2006; 17(4): 22-32.
[7] Christoph HF, Hämmerle CH, Jung RE. Systematic review of the survival of implants in bone sites augmented with barrier membranes (guided bone regeneration) in partially edentulous patients. J Clin Periodontology 2002; 29(3): 226-231.

[8] Corrente G, Abundo R, Cardaropoli D, Cardaropoli G, Martuscelli G. Long-term evaluation of osseointegrated implants in regenerated and nonregenerated bone. Int $J$ Periodontics Restor Dent 2000; 20: 390-397.

[9] Simion M, Fontana F, Rasperini G, Maiorana C. Long-term evaluation of osseointegrated implants placed in sites 
augmented with sinus floor elevation associated with vertical ridge augmentation: a retrospective study of 38 consecutive implants with 1- to 7-year follow-up. Int J Periodontics Restor Dent 2004; 24: 208-221.

[10] Clementini A, Morlupi C, Agrestini A, Barlettani C. Immediate versus delayed positioning of dental implants in guided bone regeneration or onlay graft regenerated areas: a systematic review. Int J Oral Maxillofac Surg 2013; 42: 643-650.

[11] Brunel G, Brocard D, Duffort JF, et al. Bioabsorbable materials for guided bone regeneration prior to implant placement and 7-year follow-up: Report of 14 cases. J Periodontol 2001; 72: 257-64.

[12] Simion M, Jovanovic SA, Tinti C, Benfenati SP. Long-term evaluation of osseointegrated implants inserted at the time or after vertical ridge augmentation. A retrospective study on 123 implants with 1-5 year follow-up. Clinical Oral Implants Res 2001; 12: 35-45.

[13] Buser D, Dula K, Hirt HP, Schenk RK. Ridge augmentation using autografts and barrier membranes: a clinical study with 40 partially edentulous patients. J Oral Maxillofac Surg 1996; 54(4): 420-32.

[14] Lang NP, Hämmerle CH, Bragger U, Lehmann B, Nyman SR. Guided tiasu regeneration in jaw bone defects prior to inplant placement. Clin Oral Implants Res 1994; 5: 92-97.

[15] Nevins M, Mellonig JT. Advantages of localized ridge augmentation prior to implant placement: a staged event. Int J Periodont Restor Dent 1994; 14(2): 96-111.

[16] Retzepi MI, Donos N. Guided bone regeneration: biological principle and therapeutic applications. Oral Implants Res 2010 Jun; 21(6): 567-76.

[17] Matthew V, Lian PM, Huynh B, Schoolfield J, Cochran DL. A lateral ridge augmentation study to evaluate a synthetic membrane for guided bone regeneration: an experiment in the canine mandible. Clin Oral Implants Res 2016; 27: 73-82.
[18] Ortolandi E, Quadrini F, Bellisario D, Santo L, Polimeni A, Santarsiero A.

Mechanical qualification of collagen membranes used in dentistry. Ann Ist Super Sanita 2015; 51(3): 229-235.

[19] HertenM, Jung RE, Ferrari D, Rothamel D, Golubovic V, Molenberg A, Häm Merle CH, Becker J, Schwarz F. Biodegradation of different synthetic hydrogels made of polyethylene glycol hydrogel RGD peptide modification: an immuno-histochemical study in rats. Clin Oral Implants Res 2009; 20: 116-125.

[20] Hürzeler M, Thalmair T, von Mohrenschildt S, Fickl S. Double-Layer technique for alveolar ridge augmentation: A case report. Pract Periodontics Aesthetic Dent 2009; 21:185-191.

[21] Konstantinos N, Malizos V, Loukia K, Papatheodorou J. The healing potential of the periosteum molecular aspects. Int J Care Injured 2005; 36: 13-19.

[22] Buser D, Martin W, Belser UC. Optimizing esthetics for implant restorations in the anterior maxilla: anatomic and surgical considerations. Int J Oral Maxillofac Implants 2004; 19: 43-61.

[23] Peetz M. Characterization of xenogenic bone material. In : Boyne, P.J. ed. Osseous reconstruction of the maxilla and the mandible. 1997: 87-100.

[24] Simion M, Trisi P, Piattelli A.

Vertical ridge augmentation using a membrane technique with osseointegrated implants. Int J Periodontics \& Restor Dentistry 1994; 14: 497-511.

[25] Simion M, Jovanovic SA, Trisi P, Scarano A, Piattelli A. Vertical ridge augmentation around dental implants using a membrane technique and autogenous bone or allografts in humans. Int J Periodontics \& Restor Dentistry 1998; 1 8: 8-23.

[26] Bosshard D, Schenk R. Biologic basis of bone regeneration. I $n$ : Buser D. 20 years of guided bone regeneration in implant dentistry. Quintessence edit, Chicago; 2010.
[27] Retzepi M, Donos N. Guided bone regeneration. Biological principle and therapeutic applications. Clin Oral Implants Res 2010; 21: 567-576.

[28] Broggini N, Bosshardt D, Jensen S, Borstein M, Wang C, Buser D. Bone healing around nanocrystalline hydroxyapatite, deproteinized bovine bone mineral, biphasic calcium phosphate, and autogenous bone in mandibular bone defects. Society for Biomaterials 2014: 1479-87.

[29] Karageorgiou V, Kaplan D. Porosity of 3D biomaterial scaffolds and osteogenesis. Biomaterials 2005; 26(27): 5474-91.

[30] Klawitter J, Hulber S. Application of porous ceramics for the attachement of load bearing internal orthopedic applications. J Biomed Master Res Symp 1971; 2: 161-230.

[31] Cardaropoli P, Gavegli L, Cardaropoli G. Collagen membrane, bovine bone mineral and fibrin sale. Clinical and histologic findings. Int J Periodontics Restor Dent 2013; 33; 583-589.

[32] Hellem S, Astrand P, Stenstro MB, Engquist B, Bengtsson M, Dahlgren $S$. Implant treatment in combination with lateral augmentation of the alveolar process: a 3-year prospective study. Clin Implant Dent Relat Res 2003; 5(4): 233-40.

[33] Caplanis N, Sigurdsson TJ, Rohrer MD, Wikesjo UM. Effect of allogeneic, freeze-dried, demineralized bone matrix on guided bone regeneration in supra-alveolar peri-implant defects in dogs. Int $\mathrm{J}$ Oral Maxillofac Implants 1997; 12: 634-642.

[34] Zitzmann NU, Naef R, Scharer P. Resorbable versus non-resorbable membranes in combination with Bio-Oss for guided bone regeneration. Int J Oral Maxillofac Implants 1998; 13: 576

[35] Evdokia C, Tat Fai C, Howard JD. Maintaining space in localized ridge augmentation using guided bone regeneration with tenting screw technology Biochim Biophys Acta 2013; 44(10): 37-45. 